\title{
In Vivo Characterization and Genome-based Approach to Lactobacillus Johnsonii Byun-jo-01 and Its Probiotic Properties
}

\section{Dongjun Kim}

Sungkyunkwan University

Mun-ju Cho

Sungkyunkwan University

Yongjun Lee

Sungkyunkwan University

Eui-Joon Kil

Sungkyunkwan University

\section{SungJune Byun}

Rural Development Administration

Sukchan Lee ( $\square$ sukchan107@gmail.com )

Sungkyunkwan University https://orcid.org/0000-0002-9964-0176

\section{Original Paper}

Keywords: Lactobacillus johnsonii Byun-jo-01, whole-genome sequencing, probiotics, murine norovirus, transcriptome sequencing, safety

Posted Date: February 4th, 2021

DOl: https://doi.org/10.21203/rs.3.rs-170758/v1

License: (c) (i) This work is licensed under a Creative Commons Attribution 4.0 International License. Read Full License 


\section{Abstract}

To develop new antiviral probiotics, bacteria were isolated from the microbiome in a murine intestine. In 16S rDNA sequence analysis, most isolates were identified as Lactobacillus johnsonii. These isolates were further assessed using whole-genome sequencing through the Illumina and PacBio platform, which revealed that the isolates were new strains. A novel probiotic strain, Lactobacillus johnsonii Byun-jo-01, was evaluated to determine its probiotic characteristics of safety, immune modulation, and antiviral efficacy against murine norovirus. Oral administration of L. johnsonii Byun-jo-01 was demonstrated to be safe in mice in terms of body weight, food intake, and bacterial translocation. Additionally, the expression levels of IFN-beta and IFN-gamma induced by L. johnsonii Byun-jo-01 in the small intestines of mice were higher than those in mice fed $L$. paracasei ATCC 334 and L. reuteri KACC 11452. Among the three different bacterial strains used in this study, L. johnsonii Byun-jo-01 showed the highest antiviral efficacy against murine norovirus, reducing the viral titer in fecal samples by 28 times compared with mice infected with murine norovirus. To support those in vivo experiments, genome-based data mining was performed to investigate which genes related to probiotic-specific markers were highly expressed in this isolate. Specifically, DnaK, GroEL, GroES, and GrpE, which are involved in the acid adaptation required to overcome the harsh in vivo condition, were highly expressed. Taken together, these results suggest that host-originated probiotics can be more effective than bacteria isolated from other sources, such as fermented food.

\section{Introduction}

Intestinal microbiota are involved in the host's overall immune system and have both beneficial and deleterious effects on health depending on the interactions between intestinal microorganisms and the host. Microbial diversity in the gut normally allows the gut to act as a bioreactor (Bäckhed et al. 2005). Within that healthy microbial diversity, the genus Lactobacillus is a probiotic that provides an immune boost to human beings and other animals when ingested in adequate amounts (Ishibashi and Yamazaki 2001). Despite the long clinical success of probiotic therapy, questions have been raised about its safety (Gasser 1994). Cases are very rare, but probiotic infections such as bacteremia and endocarditis mediated by Lactobacilli and Bifidobacterium in immunocompromised patients have been reported (Doron and Snydman 2015; Gouriet et al. 2012). Therefore, selecting probiotics through a rigorous screening process has been of great interest.

Noroviruses are the leading cause of viral gastroenteritis and are known worldwide to account for about 18 percent of diarrheal cases (de Graaf et al. 2016; Lopman et al. 2016). Outbreaks of human norovirus occur continuously worldwide, and infections cause vomiting and diarrhea in people of all ages (Fankhauser et al. 1998; Hansman et al. 2010; Hutson et al. 2004; Lopman et al. 2002). Despite the continued public-health emphasis on norovirus infection, effective vaccines targeting norovirus have not been developed. Because human norovirus is incompatible with cell culture systems, most research on norovirus has focused on murine norovirus, which shares characteristics with human norovirus (Kim et al. 2015; Wobus et al. 2006). 
The absence of a laboratory analysis system for human norovirus has hindered the development of antiviral therapeutics. Only a specific part of the virus, virus-encoded cysteine protease (3CLpro), has been targeted; other research has sought methods to prevent infection (Rocha-Pereira et al. 2014). Interest in norovirus therapeutics that use probiotics or recombinant probiotics from the Lactococcus and Lactobacillus genera has been increasing (Aboubakr et al. 2014; Hoang et al. 2015).

We previously reported that recombinant Lacticaseibacillus paracasei ATCC 334 expressing the 3D8 SCFV protein had antiviral effects against murine norovirus. When L. paracasei ATCC 334 expressing 3D8 ScFv was orally administered to mice, the population of the intestinal probiotic Pediococcus acidilactici was increased in their intestines, whereas the population of Helicobacterspecies decreased (Cho et al. 2018).

Even though L. paracasei ATCC 334 had an antiviral effect, this Lacticaseibacillus isolated from cheese has a fairly short retention time in animals. Because the immune enhancement effect in the host is expected to increase with the duration in the intestine, lactic acid bacteria were isolated from a host microbiome to screen for probiotics that have a high survival rate and long intestinal duration in animals.

Many previous reports have used probiotics as antiviral agents against various viruses. Most of those studies have shown that lactic acid bacteria increase a host's innate immunity and induce resistance to the virus. When Lactobacillus gasseri SBT2055 was administered to mice, the survival rate of mice infected with the H1N1 PR8 strain was increased, and the expression of antiviral genes such as myxovirus resistance 1 (Mx1) and 2'-5' oligoadenylate synthetase 1A (Oas1a) in lung tissues was upregulated (Nakayama et al. 2014). Various antiviral effects have been reported from Lactobacillus, but it is important to investigate the characteristics and antiviral mechanisms of newly isolated Lactobacillus strains because each Lactobacillus has a variety of antiviral mechanisms.

Advances in next-generation sequencing (NGS) techniques have resulted in easy availability of genomes of many Lactobacillus species (Dong et al. 2017; Inglin et al. 2017; Kazou et al. 2017; Kim et al. 2018). Safety assessment of probiotics has focused on searching for safe and/or harmful genes through in silico analysis (Wei et al. 2012; Zhang et al. 2012).

Our purpose in this research is to isolate Lactobacillus strains from a mouse microbiome and find antiviral probiotics which have strong colonization ability in mouse intestine. We used an in vivo system to assess $L$. johnsonii Byun-jo-01, a strain isolated from the small intestines of mice, to determine its antiviral efficacy, immune-boosting effect, and safety. We also performed transcriptome sequencing to supplement the in vivo data and screen for safety genes through data mining.

\section{Materials And Methods}

\section{Sample collection and isolation of bacteria}

We aseptically sampled the small intestines of specific pathogen-free ICR mice (DBL, Republic of Korea). After sampling, the extracted tissues were divided into three parts (duodenum, jejunum, ileum) and 
homogenized with stainless steel beads (NEXT ADVANCE, USA) and phosphate-buffered saline (PBS). To isolate Lactobacillus species, each part of the intestine was spread on MRS (Man-Rogosa-Sharpe) plates that were then incubated at $37^{\circ} \mathrm{C}$ for 48 hours. Each colony was selected from the plates and cultured on MRS medium. Using microscopy, bacteria with a rod shape were selected and maintained in MRS medium supplemented with $80 \%$ glycerol at $-80^{\circ} \mathrm{C}$. Another probiotic strain, Lacticaseibacillus paracasei ATCC 334, was provided by Dr. Jos Seegers (Falcobio, Netherlands), and Limosilactobacillus reuteri KACC 11452 and Lactobacillus johnsonii KCCM 32825, which were isolated from humans, were provided by the Rural Development Administration (Republic of Korea).

\section{Primer design}

All the primer sets used in this study are listed in Table 1. Previously described by Yoon et al (Kim et al. 2012), the $9 \mathrm{~F}$ and $1542 \mathrm{R}$ primers, which target bacterial $16 \mathrm{~S}$ rDNA as a universal primer, were used to identify the Lactobacillus species. The reference sequences of cytokines interferon (IFN)- $\beta$ and IFN- $\gamma$ were retrieved from GenBank (www.ncbi.nlm.nih.gov) with the following accession numbers (NM_010510.1, NM_008337.3, NM_031168.1, and NM_013693.2, respectively). Additionally, the primer set for murine norovirus detection was obtained from the sequence of the MNV capsid protein (JQ237823.1). All primers were designed using the Primer 3 program.

\section{DNA isolation}

Lactobacillus genomic DNA was prepared for bacterial identification and whole-genome sequencing. First, $200 \mu$ of bacterial seed on $4.8 \mathrm{ml}$ of MRS medium was used for DNA preparation. When the $\mathrm{OD}_{600}$ value reached 1.0 , ampicillin $(0.1 \mathrm{mg} / \mathrm{ml})$ was administered for 1 hour at $37^{\circ} \mathrm{C}$ to weaken the cell wall of the Lactobacillus. Bacterial cells were collected by centrifugation at $13,000 \times \mathrm{g}$ for $1 \mathrm{~min}$ and washed twice with PBS. Then the bacterial pellet was treated with a lysis mixture $(20 \mathrm{mg} / \mathrm{ml}$ lysozyme, $50 \mathrm{U} / \mathrm{ml}$ Mutanolysin in Tris-EDTA buffer) for 1 hour at $37^{\circ} \mathrm{C}$. Genomic DNA was isolated using a G-spin ${ }^{\mathrm{Tm}}$ Genomic DNA Extraction Kit (for Bacteria) (iNtRON Biotechnology, Republic of Korea) according to the manufacturer's protocols. The concentration of genomic DNA was measured at $260 \mathrm{~nm}$ with a spectrophotometer.

\section{Amplification of 16S rRNA}

The prepared genomic DNA was used to identify the isolated Lactobacillus by amplifying the 16S rRNA gene. Amplification of the $16 \mathrm{~S}$ rRNA amplicon was performed with the $9 \mathrm{~F}$ and $1542 \mathrm{R}$ universal bacterial primers using a thermal cycler (Bio-Rad $\mathrm{T} 100^{\mathrm{TM}}$ ). The PCR conditions were as follows: initial denaturation of DNA for $5 \mathrm{~min}$ at $95^{\circ} \mathrm{C}$, followed by 35 cycles of denaturation of DNA for $30 \mathrm{sec}$ at $95^{\circ} \mathrm{C}$, an annealing step for $30 \mathrm{sec}$ at $55^{\circ} \mathrm{C}$, an extension step for $1 \mathrm{~min} 30 \mathrm{sec}$ at $72^{\circ} \mathrm{C}$, and final extension for $10 \mathrm{~min}$ at $72^{\circ} \mathrm{C}$. PCR products were detected by electrophoresis with a $1 \%$ agarose gel stained with ethidium bromide, and target bands were visualized on a UV illuminator.

\section{Identification of Lactobacillus species}


The PCR product was purified from the gel extraction using a Zymoclean ${ }^{\mathrm{TM}}$ Gel DNA Recovery Kit (ZYMO RESEARCH, USA) according to the manufacturer's protocols. The 24-hour ligation step was performed using the $\mathrm{PGEM}{ }^{\circledR}-\mathrm{T}$ easy vector system (Promega, USA) at $16^{\circ} \mathrm{C}$. The ligation mixture was transformed to a DH5a competent cell. To select the target genes, we used the blue-white screening procedure and cultured only white colonies in Luria-Bertani broth (Difco Laboratories, USA) supplemented with 100 $\mu \mathrm{g} / \mathrm{ml}$ of ampicillin. Plasmid preparation was performed using a AccuPrep ${ }^{\circledR}$ Nano-Plus Plasmid Extraction Kit (BIONEER, Republic of Korea) according to the manufacturer instructions. The target gene was confirmed using restriction enzyme digestion (Eco RI), followed by Sanger/Capillary sequencing (Macrogen, Republic of Korea). Identification of the bacterial species was carried out using the EzTaxon-e database.

\section{Phylogenetic analysis}

The conserved 16S rRNA sequences of various Lactobacillus johnsonii reference strains and other species (L. rhamnosus GG, L. fermentum IFO 3956, and L. paracasei ATCC 334) were obtained from GenBank, including mouse isolates, to determine phylogenetic relationships. Before creation of a phylogenetic tree, we performed multiple alignments using the MEGA program (version 7). Phylogenetic relationships were identified through the Neighbor-Joining method with 1000 bootstrap replications.

\section{Whole-genome sequencing, assembly, annotation}

Whole-genome sequencing of $L$. johnsonii Byun-jo-01 was performed as previously reported (Kim et al. 2018). In brief, lysis agents were used ( $50 \mathrm{U} / \mathrm{ml}$ Mutanolysin, $20 \mathrm{mg} / \mathrm{ml}$ lysozyme) to weaken the bacterial cell wall before isolating the DNA, and then genomic DNA was prepared using a G-spin ${ }^{\mathrm{Tm}}$ Genomic DNA Extraction Kit (for Bacteria) (iNtRON Biotechnology, Republic of Korea) according to the manufacturer's protocols. Using a combination of PacBio RS II (Pacific Biosciences, USA) and Illumina Hiseq2000 (Illumina, Inc., USA), whole genome sequencing was performed at Macrogen (Seoul, Republic of Korea), resulting in a complete genome. The assembly used to construct the complete genome was a two-step process. First, with only the raw sequencing data generated from PacBio sequencing, de novo assembly was carried out using SMRT Analysis software version 2.3.0 (Zhang et al. 2000). Second, to decrease the error rate in the genome assembly and improve genome completeness, hybrid assembly using Pilon (v1.21) (Walker et al. 2014) was conducted with raw data generated from Illumina sequencing. Putative coding genes were annotated with Prodigal version 2.6 software (Hyatt et al. 2010). Using both ARAGON version 1.2 and RNAmmer version 1.2 software (Lagesen et al. 2007), tRNA and rRNA genes were identified. The default parameters were used in all software. The complete genome sequence of $L$. johnsonii Byun-jo-01 was deposited in GenBank under accession number CP029614.

\section{Comparison of circular genome}

Using whole genome information from similar L. johnsonii strains uploaded in the NCBI genome database (http://www.ncbi.nlm.nih.gov/genome), genome completeness was confirmed. Seven $L$. johnsonii strains were obtained based on phylogenetic relationships: L. johnsonii strains NCC533, N6.2, 
BS15, DPC60206, FI9785, UMNLJ21, and UMNLJ22, along with L. johnsonii Byun-jo-01. These strains were subjected to comparative analysis to identify similarities among them. Comparison of whole genomes was conducted using the BLAST Ring Image Generator (BRIG) (Alikhan et al. 2011).

\section{Mice, virus, and cell culture}

Depending on the purpose of the experiment, we used various kinds of mice. To isolate Lactobacillus, we used six-week-old, female, specific pathogen-free ICR mice (DBL, Republic of Korea). Female MNVseronegative C57BL/ 6 mice (6 weeks) were used to test the antiviral efficacy of Lactobacillus isolates against MNV-1.CR6. All mice were acclimatized for 1 week before experiments and were housed under standard laboratory conditions. All animal procedures performed in this study were reviewed, approved, and supervised by the Institutional Animal Care and Use Committee of Sungkyunkwan University (SKKUIACUC2018-10-07-3). RAW264.7 cells were maintained in Dulbecco's modified Eagle's medium (Hyclone, USA) containing 10\% heat-inactivated fetal bovine serum (Alphabioregen, USA), $100 \mathrm{units} / \mathrm{ml}$ of penicillin, and $100 \mu \mathrm{g} / \mathrm{ml}$ of streptomycin at $37^{\circ} \mathrm{C}$ in a $5 \% \mathrm{CO} 2$ incubator. Murine norovirus GV/CR6/2005/USA (MNV) was provided from Washington University by Herbert W. Virgin and grown in RAW264.7 cells (McCartney et al. 2008).

\section{Determination of survival and retention of $L$. johnsonii Byun-jo-01}

To determine the survival and retention time of L.johnsonii Byun-jo-01 in the mouse gastrointestinal tract, we modified the pSLP111.3 vector (originally provided by Dr. Jos Seegers) by replacing the xyloseinducible promoter with a lactate dehydrogenase (LDH) constitutive promoter as previously described (Oozeer et al. 2005). Transformation of the PSLP111.3 vector into L. paracasei ATCC 334 and L. johnsonii Byun-jo-01 was carried out using the electroporation method as previously described (Hoang et al. 2015). Each transformed Lactobacillus was used to differentiate the administered Lactobacillus from the other commensal bacteria in the feces of mice. The mice were assigned to four experimental groups (three mice per group): 1) presence or absence of antibiotic pretreatment before the experiment and 2) the number of feedings. To maintain the pSLP111.3 vector and remove partially commensal bacteria from the small intestine, all mice were given water containing $3 \mu \mathrm{g} / \mathrm{ml}$ chloramphenicol for $24 \mathrm{~h}$. Food and water were withdrawn for $18 \mathrm{~h}$ before the feeding trials. The mice were fed $10^{8} \mathrm{CFU}$ of $L$. paracasei ATCC 334 or L. johnsonii Byun-jo-01 once or 3 times every 2 days using feeding needles (20 gauge). In the experimental group that did not receive antibiotics, Lactobacillus was administered directly without pretreatment. Fecal samples were collected from each mouse from day 1 to day 11 following the first administration of Lactobacillus. At each time point, the fecal samples were weighed and then homogenized in PBS, followed by serial dilution. Diluted fecal samples were inoculated on MRS plates containing $3 \mu \mathrm{g} / \mathrm{ml}$ of chloramphenicol. After anaerobic incubation at $37^{\circ} \mathrm{C}$ for 2 days, the viable counts in the fecal samples were determined, and the number of colony forming units (CFUs) of Lactobacillus per gram of feces was calculated. Negative control mice were fed only PBS.

\section{Bacterial translocation}


Translocation events, in which administered bacteria move from gut tissues to other organs, were confirmed as previously described (Nguyen et al. 2007). The liver and kidney were harvested aseptically, and each organ was homogenized in PBS using stainless steel beads (NEXT ADVANCE, USA). About 100 $\mu \mathrm{l}$ of homogenates and $20 \mu \mathrm{l}$ of blood were inoculated on MRS agar and BHI (Brain Heart Infusion) agar (BD Biosciences, USA), and the MRS plates were incubated for 48 hours at $37^{\circ} \mathrm{C}$. After 48 hours, incidence of translocation was expressed as number of mice in which bacteria were detected / number of total mice. We defined occurrence of an event as one or more colonies on the agar plates.

\section{Immune-boosting mediated by L. johnsonii Byun-jo-01}

Total RNA was prepared from small intestine tissues using TRI reagent (Molecular Research Center, Inc., USA). RNA concentrations were measured at $260 \mathrm{~nm}$ absorbance using a spectrophotometer. cDNA was synthesized from $5 \mu \mathrm{g}$ of total RNA using Moloney murine leukemia virus reverse transcriptase (Bioneer, Republic of Korea) according to the manufacturer's protocols. The following cytokines were quantitatively measured to compare expression levels induced by Lactobacillus: IFN- $\beta$ and IFN- $\gamma$. Quantitative real-time PCR was conducted using SYBR Premix Ex Taq (TaKaRa, Japan) and a Rotor-Gene Q system (Qiagen, Australia). Data were analyzed using Rotor-Gene Q series software version 2.3.1 (Qiagen, Australia).

\section{Antiviral efficacy against MNV CR6}

In addition to the L. paracasei ATCC 334 strain, the antiviral activity test was performed using $L$. johnsonii KCCM 32825, which originated from a human, as a control probiotic. After administration of $10^{8} \mathrm{CFU}$ of Lactobacillus (L. paracasei ATCC 334, L. johnsonii KCCM 32825, or L. johnsonii Byun-jo-01) to the mice, $10^{5} \mathrm{PFU}$ of MNV was given orally. Feces were collected on days $5,7,9$, and 14 after viral infection. Fecal RNA was extracted using a Quick-RNATM Fecal / Soil Microbe Microprep kit (ZYMORESEARCH, USA). cDNA was synthesized from $5 \mu \mathrm{g}$ of total RNA using CellScript cDNA Synthesis Master Mix (CellSafe, Republic of Korea) with a random primer. The viral titer was analyzed among experimental groups using quantitative real-time PCR.

\section{Transcriptome sequencing}

L. johnsonii Byun-jo-01 grown in pH4.8 conditions to mimic its niche was used for RNA preparation with a NucleoSpin ${ }^{\circledR}$ RNA kit (MACHEREY-NAGEL, Germany). To prevent DNA contamination in the total RNA samples, DNase was added, and then the purified mRNA was isolated using Ribo-Zero rRNA Removal Kit (Bacteria) (Illumina, USA), completely removing the rRNA from the samples. The cDNA library was constructed with a TruSeq RNA Sample Prep Kit v2 for library preparation according to the manufacturer's instructions. Paired-end libraries (200-400bp insert sizes) were sequenced using the HiSeq2000 platform (Macrogen, Republic of Korea).

\section{Mapping and Fragments Per Kilobase of transcript per Million (FPKM) calculation}


The raw data (4 fastq files) generated from transcriptome sequencing were deposited in GenBank under accession number SRR9001782. Adaptor sequences were removed by Trimmomatic 0.32 software, and then only the trimmed reads were mapped onto the reference sequence (the genome of L. johnsonii Byunjo-01) using BBMap (short-read aligner) (Bushnell 2014). Default parameters were used for all software during this process. Probiotic-related markers were searched using data mining and previously published papers.

\section{Statistical analysis}

All analyses were carried out in triplicate and assessed using GraphPad Prism (GraphPad software version 5). Data are presented as means \pm SEM. $P<0.05$ was considered significant. One-way analysis of variance (ANOVA) was used for statistical analysis.

\section{Results}

\section{Identification of Lactobacillus isolates from mouse gastrointestinal tract}

The divided tissues (duodenum, jejunum, ileum) of mice were used to find probiotic candidates. MRS agar plates were used to specifically isolate Lactobacillus from intestinal tissues. Viable bacterial colonies were selected for identification using universal 16S rRNA primers as previously described (Kim et al. 2012). Most isolates were found to be L. johnsonii, except for a few species (Lactobacillus intestinalis, putative Lacticaseibacillus paracasei), and this isolate showed $100 \%$ similarity with various $L$. johnsonii strains including $L$. johnsonii N6.2 and $L$. johnsonii DPC 6026. The $L$. johnsonii isolates finally selected for further experiments were isolated in the jejunum, the part of the small intestine between the duodenum and ileum.

\section{Phylogenetic relationships based on 16S rRNA of $L$. johnsonii strains}

To determine phylogenetic relationships between the isolated strain and previously reported similar strains, a phylogenetic tree was created using 16S rDNA sequences, which are known to be conserved in microorganisms. The sequence data for the reference strains, L. fermentum, L. brevis, L. plantarum, $L$. salivarius, L. paracasei, and L. crispatus, and various L. johnsonii stains were retrieved from the NCBI database (www.ncbi.nlm.nih.gov). Sequence alignment using 16S rRNA (average 1500bp) was carried out using the CLUSTAL X program, followed by construction of a phylogenetic tree based on the Neighbor-Joining method with 1000 bootstrap replicates. The result, as shown in Fig. 1, indicates that the $L$. johnsonii mouse isolates belong to same clade as various other $L$. johnsonii strains.

\section{Genomic characterization of L. johnsonii Byun-jo-01}

The 16S rRNA gene is a standard for bacterial identification because of its presence in all bacteria. However, it has been suggested that basing the identification of bacterial strains on only the 16S rRNA gene is limiting (Stackebrandt 2006). To further identify this isolate, therefore, we conducted wholegenome sequencing using both the Illumina Hiseq 2000 (2 x 100 bp paired-end sequencing; Illumina, Inc., 
USA) and PacBio RS II (Pacific Bioscience, USA) platforms. General genome features and a circular map of the chromosome of L. johnsonii Byun-jo-01 are shown in Table 2 and Fig. 2, respectively. According to the genome features table, single-molecule real-time (SMRT) cell sequencing with 20-kb inserts yielded $1,847,047,342$ bp (182,640-bp-long reads). Draft genome assembly was carried out using 182,640 reads generated from PacBio sequencing. To improve the accuracy of genome assembly, a total of $81,648,222$ short reads generated from the lllumina sequencing was used for hybrid assembly. That two-step assembly process generated a complete genome with a size of 1,959,519 bp (N50 values 1,959,519) and a GC content of $34.7 \%$. The annotation process indicated that the genome of $L$. johnsonii Byun-jo-01 consists of 1,781 coding sequences, 21 rRNAs, and 77 tRNAs. As shown in Fig. 3, the distribution of clusters of orthologous groups (COG) in L. johnsonii Byun-jo-01 was identified. Among the COG functional categories, J (translation, ribosomal structure, and biogenesis), G (carbohydrate transport and metabolism), M (cell wall/membrane/envelope biogenesis), and $\mathrm{K}$ (translation) were the most abundant in the genome.

In addition to the 16S rRNA sequences, bacterial identification was carried out on a whole-genome level, and $L$. johnsonii Byun-jo-01 was found to be a novel strain. For identification, similar L. johnsonii strains (L. johnsonii NCC 533, L. johnsonii strain BS15, L. johnsonii N6.2, L. johnsonii strain UMNLJ22, L. johnsonii strain UMNLJ21, and L. johnsonii DPC6026) were retrieved from the NCBI genome database. To visualize the similarity in the whole genomes of the $L$. johnsonii strains, BRIG was used. The overall similarity of the genomes is shown in Fig. 4. Using BLAST, the overall similarity of the L. johnsonii genome with the reference strain was numerically compared and found to be greater than $98 \%$ (Table 3).

\section{Survival and retention of $L$. johnsonii Byun-jo-01 in the gastrointestinal tract}

The ability of $L$. johnsonii Byun-jo-01 to reach and survive in the gastrointestinal tract of mice was evaluated by counting viable colonies of $L$. johnsonii Byun-jo-01 in fecal samples after oral administration under various antibiotic treatments and numbers of feeding trials. To distinguish between administered bacteria and other intestinal bacteria in the fecal samples, $L$. paracasei ATCC 334 and $L$. johnsonii Byun-jo-01 were transformed using the pSLP111.3 expression vector containing a chloramphenicol-resistant gene and LDH constitutive promoter (Hoang et al. 2015), and those transformed bacteria were used as the selection tool instead of wild type Lactobacillus. For all the Lactobacillus used in the experiment, $10^{8} \mathrm{CFUs}$ were administered via oral administration. The survivability of $L$. johnsonii Byun-jo-01 over time after administration is shown in Fig. 5 . To facilitate placement of the bacteria in the intestine, commensal bacteria were removed by giving the mice water containing chloramphenicol. As expected, all administered bacteria were detected in the feces under any experimental condition. In the mice fed antibiotics, L. johnsonii Byun-jo-01 was present in the feces $24 \mathrm{~h}$ after administration, reaching a level of $1 \times 10^{6} \mathrm{CFU} / \mathrm{g}$, and that level decreased gradually over time. Unlike L. paracasei ATCC 334, L. johnsonii Byun-jo-01 survived for about 11 days after both single and triple doses (Figs. 5aand 5b). However, without antibiotics, the number of CFUs $24 \mathrm{~h}$ after administration was relatively small, and the duration of $L$. johnsonii Byun-jo-01 was much shorter than in the antibiotics 
condition (Figs. 5c and 5d). The difference between these results indicates that the antibiotics allowed $L$. johnsonii Byun-jo-01 to establish and remain in the intestine for a long time.

\section{Safety assessment of L. johnsonii Byun-jo-01}

Mice were given $L$. johnsonii Byun-jo-01 at a dosage of $10^{8} \mathrm{CFU} /$ mouse daily for 2 weeks to discern any safety issues. After administration of Lactobacillus, changes in body weight, food intake, and organ weight were determined. Prior to the experiment, all subjects started at the same body weight. During the experiment, no abnormal behavior was observed in any experimental group compared with the control mice. The body weight increased slightly in all experimental groups over time (Fig. 6), but none of the changes were statistically significant. Additionally, no detectable changes in food intake were observed, as shown in Table 3. Food intake was measured every 2 days because daily intake varied widely among individuals. The weight and appearance of the liver, small intestine, and spleen were confirmed after sacrifice. There was no significant change in organ weight between the Lactobacillus-treated mice and the negative control mice, and no significant lesions were found (Fig. 7). Taken together, these findings suggest that the safety of $L$. johnsonii Byun-jo-01 did not differ significantly from that of control $L$. paracasei ATCC 334 when used as a probiotic.

\section{Analysis of bacterial translocation}

Even though the safety test found no abnormalities, as described above, the bacterial translocation test is necessary before a strain can be developed and used as a probiotic. Bacterial translocation can occur when the balance of the intestinal microbiome is disrupted and the junction of intestinal epithelial cells is weakened by any bacteria. This allows bacteria present in the intestine to migrate from the gastrointestinal tract to sites such as the spleen and bloodstream (Berg 1995). We judged translocation to have occurred if even a single colony was found on the MRS or BHI agar plates. In both the negative control mice and the L. johnsonii Byun-jo-01-fed mice, no events were detected at any site, as shown in Table 4. Only one event occurred in the liver of a mouse treated with L. paracasei ATCC 334. There were no significant differences in translocation incidence between the control mice and the L. johnsonii Byunjo-01-fed mice. Translocated bacteria in the liver can cause damage and increase the levels of alanine aminotransferase (ALT) and aspartate aminotransferase (AST) (Tian et al. 2015). Although we found no bacterial colonies on the plates from the organs, we performed an additional analysis to determine whether the serum ALT and AST levels were within the normal ranges. All tested mice had AST and ALT levels within the normal ranges (AST: 54-298 U/L, ALT: 17-77 U/L). The AST level in mouse serum was $110 \mathrm{U} / \mathrm{L}$ in control mice, $105 \mathrm{U} / \mathrm{L}$ in L. paracasei ATCC 334-treated mice, and $109 \mathrm{U} / \mathrm{L}$ in L. johnsonii Byun-jo-01-treated mice. The ALT level was 38, 44, and $38(\mathrm{U} / \mathrm{L})$, respectively. Taken together, these results imply that $L$. johnsonii Byun-jo-01 isolated from murine intestines has no safety issues as a probiotic in mice.

\section{Immune-boosting activity of $L$. johnsonii Byun-jo-01}


Intestinal microbiota form an immunological barrier in the host and play an important role in maintaining the intestinal immune system through interactions among themselves and between intestinal microorganisms and intestinal immune cells (Delcenserie et al. 2008). Lactobacillus, as reported in many references in which lactic acid bacteria were applied to animal models for clinical trials, are particularly known as health-promoting factors (Galdeano and Perdigon 2006b; Gill et al. 2000; Hori et al. 2002). Lactobacillus strains regulate host immunity and induce secretion of various cytokines in different ways, depending on the type of Lactobacillus (Delcenserie et al. 2008). To determine how L. johnsonii Byun-jo01 enhances intestinal immunity, we evaluated cytokines: IFN- $\beta$ and IFN- - . To determine how expression of the selected cytokines was increased by the administered strains (L. paracasei ATCC 334, L. reuteri KACC 11452, and L. johnsonii Byun-jo-01), a dose of $10^{8} \mathrm{CFU}$ of Lactobacillus was administered to mice daily for 2 weeks, as in the safety assessment, and the cytokine profiles in intestinal tissue were examined using quantitative real-time PCR. We expected that the lactic acid bacteria isolated from the host microbiome would confer a greater immune enhancement on the original host, so we used humanderived $L$. reuteri KACC 11452 as another control group. Compared with the negative control mice, as shown in Fig. 8, the relative mRNA expression of IFN- $\beta$ and IFN- $\gamma$ in mice treated with $L$. johnsonii Byun-jo01 was about 9.04-fold and 23-fold higher, respectively. The expression levels of IFN- $\beta$ and IFN- $\gamma$ mediated by L. johnsonii Byun-jo-01 were 3.82 and 6.39 times higher, respectively, than those mediated by L. paracasei ATCC 334.

\section{Antiviral activity test of $L$. johnsonii Byun-jo-01 against murine norovirus}

The C57BL/ 6 mouse model was chosen for this experiment because it is particularly feasible for murine norovirus infection (Tomov et al. 2013). The most important factor required to control murine norovirus infection is innate immunity, involving proteins such as STAT-1, which is a primary mediator of both type I and type II interferon responses (Platanias 2005; Wobus et al. 2006). As illustrated in Fig. 8, L. johnsonii Byun-jo-01 increased the expression of IFN- $\beta$ and IFN- $\gamma$, thus conferring anti-viral efficacy to mice. To confirm whether Lactobacillus isolates protected MNV-infected mice, oral gavage was performed for 2 weeks prior to virus infection. Because the MNV CR6 strain remains chronically in vivo, no viral capsid mRNA was detected in the stool until 3 days after infection (data not shown). Using feces collected on days $5,7,9$, and 14 after viral infection, we analyzed the antiviral effects of the Lactobacillus isolates over time (Fig. 9). On the $7^{\text {th }}$ day after infection, viral capsid mRNA was maximally expressed; compared with negative control mice on day 7 , the number of viral genes in the feces of mice that received $L$. johnsonii Byun-jo-01 decreased more than 80-fold. Additionally, L. johnsonii KCCM 32825 isolated from humans was used to compare its efficacy with that of the murine strains isolated from the host microbiome. L. johnsonii Byun-jo-01 induced the highest anti-viral state in mice, 13 times better than that from the $L$. johnsonii human isolates. The most striking finding of this experiment was that the efficacy of the isolated Lactobacillus was far superior to that of Lactobacillus species isolated from other sources, such as fermented foods.

\section{Characterization of probiotic-related markers using transcriptome sequencing}


To investigate the probiotic-related genes expressed in its original niches, L. johnsonii Byun-jo-01 was cultured in MRS media at $\mathrm{pH} 4.8$, which mimics intestinal $\mathrm{pH}$ conditions, and total RNA was extracted. RNA, with the DNA completely removed, was used to purify mRNA, followed by transcriptome sequencing using the Hiseq2000 platform. Total reads (26,701,034 bp and 29,291,286 bp) were generated, and adaptor sequences were removed using Trimmomatic 0.32 software in the default condition for exact mapping. Only processed reads were used for the mapping procedure through BBMap, followed by screening of the probiotic-related genes to support our in vivo safety data. On the basis of published literature (Lebeer et al. 2008; Papadimitriou et al. 2015), we searched and analyzed common probioticrelated markers to determine their expression levels. In the definition of probiotics, virulence factors, a probiotic factor and an adaptation factor, are essential to the host-microbe interaction (Lebeer et al. 2008). The genes involved in the stress response, DnaK, DnaJ, GroES, GrpE, and the FOF1 ATP synthase subunits, were discovered, and the FPKM value of those transcripts was greater than 20,000 (Fig. 10). Additionally, genome analysis revealed many adhesion-related factors, such as sortase, dltD (Dadenylation of LTA), hemolysin III, and fibronectin-binding protein, which are responsible for adhesion to the extracellular matrix of epithelial cells (Azcarate-Peril et al. 2008). The most characterized probiotic marker, exopolysaccharide, was detected, but its expression level was much lower than that of the other markers. Overall, the expression levels of the markers involved in the stress response were up-regulated upon exposure to bile acid or gastric juice and were significantly higher than the markers involved in adhesion.

\section{Discussion}

Lactobacillus compose much of the intestinal diversity in domestic animals and are widely used as probiotics. Probiotics, commonly known to be safe, have been used in a variety of pharmaceutical drugs as well as dairy products after numerous clinical trials (Salminen et al. 1998). Nonetheless, rare cases of infection by Lactobacillus and Bifidobacterium have been reported, such as bacteremia and endocarditis in immunocompromised patients (Salminen et al. 1998). Therefore, in developing new probiotic treatments, probiotics must first be scientifically verified and undergo safety evaluations, rather than being applied directly to clinical trials (Borriello et al. 2003).

Development of NGS techniques has allowed easy access to genomic information about many microbes. Therefore, it is unnecessary to prove the safety of each probiotic experimentally in in vitro and in vivo systems. The databases can demonstrate the safety of a probiotic by enabling researchers to explore probiotic-specific genes and safety-related genes through data mining, which is much cheaper and more efficient than experimental methods (Senan et al. 2015). Thus, we attempted to prove the safety of a probiotic by combining in vivo and in silico systems in a thorough screening and thereby provide a new framework for safety evaluation.

Based on the criteria for probiotic selection, we identified isolated Lactobacillus at the genus, species, and strain levels using $16 \mathrm{~S}$ rDNA sequencing and whole-genome sequencing through NGS technology. By 
comparing similarities with strains reported previously, we found that our isolate was a novel strain, $L$. johnsonii Byun-jo-01 (Figs. 1-2, Tables 2-3).

Oral toxicity was evaluated by administering a high dose of the potential probiotic strain L. johnsonii Byun-jo-01 to mice for 2 weeks. As a result, we found no adverse effects to body weight or food intakes compared to normal mice, indicating that the potential probiotic did not show oral toxicity or have any detrimental effect on the health of mice (Table 4, Figs. 6-7). These results suggest that administration of this potential probiotic has no deleterious effects (Lara-Villoslada et al. 2009).

Indigenous bacteria can move from the gut to other tissues in an opportunistic infection, which produces toxicity markers and can cause side effects (Steffen and Berg 1983). No bacterial translocation of the potential probiotics tested here was observed in mice, except in one case treated with L. paracasei ATCC 334 (Table 5). In terms of safety assessment, the evaluation factors described above differed little from those of normal mice, confirming that administration of the selected Lactobacillus had no effect on the health of mice.

A survival-rate experiment was conducted to determine whether the mouse strain administered could reach the intestine despite exposure to gastric acid or bile acid, and the results showed a longer retention time than the control probiotic strain in all experimental conditions (Fig. 5). In particular, when given along with antibiotics, the Byun-jo-01 strain survived in the gastrointestinal tract much longer than the ATCC 334 strain, indicating that microbial reduction by antibiotics treatment helped the administered Lactobacillus colonize the gut (Hill et al. 2010). In the literature, the retention time of Lactobacillus isolated from other sources was relatively short (Su et al. 2007), whereas the mouse strain in this study originated from a mouse microbiome and was superior in the original host (mice).

Lactobacilli can lead to both innate and adaptive immunity by binding in specific patterns, microbeassociated molecular patterns (MAMPs), with pattern recognition receptors in immune cells (Abreu 2010; Kawai and Akira 2010; Wells et al. 2010). Induction of cytokines and chemokines after this binding can vary according to the combination of MAMPs and Toll-like receptors (Abreu 2010; Kawai and Akira 2010). According to many researchers, probiotics such as L. casei, L. rhamnosus, and L. plantarum can elicit different immunomodulatory effects in a strain-specific manner (Vitini et al. 2000). In vivo studies demonstrated that $L$. casei, L. rhamnosus, and L. plantarum affect both systemic and mucosal immunity by stimulating immune cells in the intestine and binding antigen-presenting cells, followed by expression of inflammatory (TNF-a, IFN- $y, \mathrm{IL}-12$ ) and regulatory cytokines (IL-4, IL-10) (Galdeano and Perdigon 2006a). Although we confirmed that intestinal immunity was increased by the Byun-jo-01 strain, especially by an increase in cytokines associated with antiviral defense such as IFN- $\beta$ and IFN- $\gamma$ (Fig. 8), the mechanism by which this strain affects the gut-associated immune system should be further elucidated.

To analyze the antiviral efficacy of the enhanced immunity, we verified the effects of the Byun-jo-01 strain using an MNV-infected mouse model. Because MNV strain CR6 is not acute but has enteric persistence (Nice et al. 2015), we demonstrated the preventive effect of the Byun-jo-01 strain against MNV from the 
$3^{\text {rd }}$ day post-infection. Compared with the control mice (MNV-infection only), the lowest level of virus titer was found in mice treated with the Byun-jo-01 strain over the entire period, and the Byun-jo-01 strain was more effective than $L$. johnsonii KCCM 32825 . Our findings in this study suggest that the benefits derived from the original host produced anti-viral efficacy because the safety and effective immune enhancement of the Byun-jo-01 strain were better than those of any other probiotics tested.

To characterize the potential probiotics validated in the in vivo system and to support the results of the preceding experiments, we conducted both whole-genome sequencing and transcriptome sequencing analysis to detect probiotic-specific genes. The two main categories used to describe probiotics are called adaptation factors and probiotic factors; in combination, they are described as virulence factors (Lebeer et al. 2008). We detected many virulence factors, such as dltD, sortase, and hemolysin III, involved in adherence in the gut. Among them, dltD was most highly expressed in the Byun-jo-01 strain (Fig. 10). Additionally, stress-related genes involved in acid adaptation (long-term acid stress resistance), DnaK, DnaJ, GroEL, GroES, and FOF1 ATP synthase subunits, were highly expressed. According to previous research, two specific proteins of $L$. johnsonii NCC 533, elongation factor Tu (EF-Tu) and the heat shock protein GroEL, are adhesive factors (Bergonzelli et al. 2006; Granato et al. 2004). Our in silico findings confirm expression of genes that can play a role as potential probiotics. These results are supportive of our in vivo findings.

Taken together, our results can be used as a foundation for the basic process of more thorough screening of potential probiotics.

\section{Declarations}

\section{Funding}

This work was supported by a grant from the Next-Generation BioGreen 21 Program (Project No. PJ01328302), Rural Development Administration, Republic of Korea.

\section{Conflicts of interest}

The authors declare no competing interests.

\section{Availability of data and material}

The complete genome of L. johnsonii Byun-jo-01 has been deposited at GenBank under the accession number CP029614. The transcriptome data generated from Illumina sequencing were deposited in Genbank under the following accession number (SRR9001782).

\section{Author's contributions}

D.K., M.-J.C., E.-J.K., S.L., S.J.B. designed the experiment and concepts. D.K., M.-J.C. performed the experiments and analyzed the data with E.-J.K. Manuscript was drafted by D.K., E.-J.K., S.L. 
D.K., M.-J.C., E.-J.K., S.L. performed analyses and interpretation of data. All authors discussed the results and commented on the manuscript.

\section{References}

Aboubakr HA, El-Banna AA, Youssef MM, Al-Sohaimy SA, Goyal SM (2014) Antiviral effects of Lactococcus lactis on feline calicivirus, a human norovirus surrogate Food and environmental virology 6:282-289

Abreu MT (2010) Toll-like receptor signalling in the intestinal epithelium: how bacterial recognition shapes intestinal function Nature Reviews Immunology 10:131

Alikhan N-F, Petty NK, Zakour NLB, Beatson SA (2011) BLAST Ring Image Generator (BRIG): simple prokaryote genome comparisons BMC genomics 12:402

Azcarate-Peril MA et al. (2008) Analysis of the genome sequence of Lactobacillus gasseri ATCC 33323 reveals the molecular basis of an autochthonous intestinal organism Appl Environ Microbiol 74:46104625

Bäckhed F, Ley RE, Sonnenburg JL, Peterson DA, Gordon JI (2005) Host-bacterial mutualism in the human intestine science 307:1915-1920

Berg RD (1995) Bacterial translocation from the gastrointestinal tract Trends in microbiology 3:149-154

Bergonzelli GE, Granato D, Pridmore RD, Marvin-Guy LF, Donnicola D, Corthésy-Theulaz IE (2006) GroEL of Lactobacillus johnsonii La1 (NCC 533) is cell surface associated: potential role in interactions with the host and the gastric pathogen Helicobacter pylori Infection and immunity 74:425-434

Borriello S, Hammes W, Holzapfel W, Marteau P, Schrezenmeir J, Vaara M, Valtonen V (2003) Safety of probiotics that contain lactobacilli or bifidobacteria Clinical infectious diseases 36:775-780

Bushnell B (2014) BBMap: a fast, accurate, splice-aware aligner. Lawrence Berkeley National Lab.(LBNL), Berkeley, CA (United States),

Cho S et al. (2018) Probiotic Lactobacillus paracasei expressing a nucleic acid-hydrolyzing minibody (3D8 ScFv) enhances probiotic activities in mice intestine as revealed by metagenomic analyses Genes $9: 276$

de Graaf M, van Beek J, Koopmans MP (2016) Human norovirus transmission and evolution in a changing world Nature Reviews Microbiology 14:421

Delcenserie V, Martel D, Lamoureux M, Amiot J, Boutin Y, Roy D (2008) Immunomodulatory effects of probiotics in the intestinal tract Current issues in molecular biology 10:37 
Dong Q-Q et al. (2017) Complete genome sequence of Lactobacillus plantarum CGMCC 8198 Genome Announc 5:e01559-01516

Doron S, Snydman DR (2015) Risk and safety of probiotics Clinical Infectious Diseases 60:S129-S134 Fankhauser RL, Noel JS, Monroe SS, Ando T, Glass RI (1998) Molecular epidemiology of "Norwalk-like viruses" in outbreaks of gastroenteritis in the United States The Journal of infectious diseases 178:15711578

Galdeano CM, Perdigon G (2006) The probiotic bacterium Lactobacillus casei induces activation of the gut mucosal immune system through innate immunity Clinical and Vaccine Immunology 13:219-226

Gasser F (1994) Safety of lactic acid bacteria and heir occurrence in human clinical infections Bull Inst Pasteur 92:45-67

Gill HS, Rutherfurd KJ, Prasad J, Gopal PK (2000) Enhancement of natural and acquired immunity by Lactobacillus rhamnosus (HN001), Lactobacillus acidophilus (HN017) and Bifidobacterium lactis (HN019) British Journal of Nutrition 83:167-176

Gouriet F, Million M, Henri M, Fournier P-E, Raoult D (2012) Lactobacillus rhamnosus bacteremia: an emerging clinical entity European journal of clinical microbiology \& infectious diseases 31:2469-2480 Granato D, Bergonzelli GE, Pridmore RD, Marvin L, Rouvet M, Corthésy-Theulaz IE (2004) Cell surfaceassociated elongation factor Tu mediates the attachment of Lactobacillus johnsonii NCC533 (La1) to human intestinal cells and mucins Infection and immunity 72:2160-2169

Hansman GS, Jiang XJ, Green KY (2010) Caliciviruses: molecular and cellular virology. Horizon Scientific Press,

Hill DA et al. (2010) Metagenomic analyses reveal antibiotic-induced temporal and spatial changes in intestinal microbiota with associated alterations in immune cell homeostasis Mucosal immunology $3: 148$

Hoang PM, Cho S, Kim KE, Byun SJ, Lee T-K, Lee S (2015) Development of Lactobacillus paracasei harboring nucleic acid-hydrolyzing 3D $8 \mathrm{scFv}$ as a preventive probiotic against murine norovirus infection Applied microbiology and biotechnology 99:2793-2803

Hori T, Kiyoshima J, Shida K, Yasui H (2002) Augmentation of cellular immunity and reduction of influenza virus titer in aged mice fed Lactobacillus casei strain Shirota Clin Diagn Lab Immunol 9:105108

Hutson AM, Atmar RL, Estes MK (2004) Norovirus disease: changing epidemiology and host susceptibility factors Trends in microbiology 12:279-287 
Hyatt D, Chen G-L, LoCascio PF, Land ML, Larimer FW, Hauser LJ (2010) Prodigal: prokaryotic gene recognition and translation initiation site identification $B M C$ bioinformatics 11:119

Inglin RC, Meile L, Klumpp J, Stevens MJ (2017) Complete and assembled genome sequence of Lactobacillus plantarum RI-113 isolated from salami Genome announcements 5

Ishibashi N, Yamazaki S (2001) Probiotics and safety- The American journal of clinical nutrition 73:465s470 s

Kawai T, Akira S (2010) The role of pattern-recognition receptors in innate immunity: update on Toll-like receptors Nature immunology 11:373

Kazou M, Alexandraki V, Pot B, Tsakalidou E, Papadimitriou K (2017) Complete genome sequence of the dairy isolate Lactobacillus acidipiscis ACA-DC 1533 Genome Announc 5:e01533-01516

Kim D, Cho M-j, Cho S, Lee Y, Byun SJ, Lee S (2018) Complete Genome Sequence of Lactobacillus johnsonii Strain Byun-jo-01, Isolated from the Murine Gastrointestinal Tract Microbiol Resour Announc 7:e00985-00918

Kim 0-S et al. (2012) Introducing EzTaxon-e: a prokaryotic 16S rRNA gene sequence database with phylotypes that represent uncultured species International journal of systematic and evolutionary microbiology 62:716-721

Kim Y, Galasiti Kankanamalage AC, Chang K-0, Groutas WC (2015) Recent Advances in the Discovery of Norovirus Therapeutics: Miniperspective Journal of medicinal chemistry 58:9438-9450

Lagesen K, Hallin P, Rødland EA, Stærfeldt H-H, Rognes T, Ussery DW (2007) RNAmmer: consistent and rapid annotation of ribosomal RNA genes Nucleic acids research 35:3100-3108

Lara-Villoslada F, Sierra S, Díaz-Ropero MP, Rodríguez JM, Xaus J, Olivares M (2009) Safety assessment of Lactobacillus fermentum CECT5716, a probiotic strain isolated from human milk Journal of dairy research 76:216-221

Lebeer S, Vanderleyden J, De Keersmaecker SC (2008) Genes and molecules of lactobacilli supporting probiotic action Microbiol Mol Biol Rev 72:728-764

Lopman B, Brown D, Koopmans M (2002) Human caliciviruses in Europe Journal of Clinical Virology 24:137-160

Lopman BA, Steele D, Kirkwood CD, Parashar UD (2016) The vast and varied global burden of norovirus: prospects for prevention and control PLoS medicine 13:e1001999

McCartney SA, Thackray LB, Gitlin L, Gilfillan S, Virgin IV HW, Colonna M (2008) MDA-5 recognition of a murine norovirus PLoS pathogens 4:e1000108 
Nakayama Y et al. (2014) Oral administration of Lactobacillus gasseri SBT2055 is effective for preventing influenza in mice Scientific reports 4:4638

Nguyen T, Kang J, Lee M (2007) Characterization of Lactobacillus plantarum PH04, a potential probiotic bacterium with cholesterol-lowering effects International journal of food microbiology 113:358-361

Nice TJ et al. (2015) Interferon- $\lambda$ cures persistent murine norovirus infection in the absence of adaptive immunity Science 347:269-273

Oozeer R, Furet J, Goupil-Feuillerat N, Anba J, Mengaud J, Corthier G (2005) Differential activities of four Lactobacillus casei promoters during bacterial transit through the gastrointestinal tracts of humanmicrobiota-associated mice Applied and environmental microbiology 71:1356-1363

Papadimitriou K, Zoumpopoulou G, Foligné B, Alexandraki V, Kazou M, Pot B, Tsakalidou E (2015) Discovering probiotic microorganisms: in vitro, in vivo, genetic and omics approaches Frontiers in microbiology 6:58

Platanias LC (2005) Mechanisms of type---and type-II-interferon-mediated signalling Nature Reviews Immunology 5:375

Rocha-Pereira J, Nascimento M, Ma Q, Hilgenfeld R, Neyts J, Jochmans D (2014) The enterovirus protease inhibitor rupintrivir exerts cross-genotypic anti-norovirus activity and clears cells from the norovirus replicon Antimicrobial agents and chemotherapy 58:4675-4681

Salminen S et al. (1998) Demonstration of safety of probiotics-a review International journal of food microbiology 44:93-106

Senan S, Prajapati J, Joshi C (2015) Feasibility of genome-wide screening for biosafety assessment of probiotics: a case study of Lactobacillus helveticus MTCC 5463 Probiotics and antimicrobial proteins 7:249-258

Stackebrandt E (2006) Taxonomic parameters revisited: tarnished gold standards Microbiol Today 33:152-155

Steffen E, Berg R (1983) Relationship between cecal population levels of indigenous bacteria and translocation to the mesenteric lymph nodes Infection and Immunity 39:1252-1259

Su P, Henriksson A, Mitchell H (2007) Survival and retention of the probiotic Lactobacillus casei LAFTI® L26 in the gastrointestinal tract of the mouse Letters in applied microbiology 44:120-125

Tian F et al. (2015) Lactobacillus rhamnosus CCFM1107 treatment ameliorates alcohol-induced liver injury in a mouse model of chronic alcohol feeding Journal of Microbiology 53:856-863 
Tomov VT et al. (2013) Persistent enteric murine norovirus infection is associated with functionally suboptimal virus-specific CD8 T cell responses Journal of virology 87:7015-7031

Vitini E, Alvarez S, Medina M, Medici M, Perdigón G (2000) Gut mucosal immunostimulation by lactic acid bacteria Biocell: official journal of the Sociedades Latinoamericanas de Microscopia Electronica et al 24:223-232

Walker BJ et al. (2014) Pilon: an integrated tool for comprehensive microbial variant detection and genome assembly improvement PloS one 9:e112963

Wei Y-X, Zhang Z-Y, Liu C, Malakar PK, Guo X-K (2012) Safety assessment of Bifidobacterium longum JDM301 based on complete genome sequences World Journal of Gastroenterology: WJG 18:479

Wells JM, Loonen LM, Karczewski JM (2010) The role of innate signaling in the homeostasis of tolerance and immunity in the intestine International Journal of Medical Microbiology 300:41-48

Wobus CE, Thackray LB, Virgin HW (2006) Murine norovirus: a model system to study norovirus biology and pathogenesis Journal of virology 80:5104-5112

Yoon J-H, Lee ST, Park Y-H (1998) Inter-and intraspecific phylogenetic analysis of the genus Nocardioides and related taxa based on $16 \mathrm{~S}$ rDNA sequences International Journal of Systematic and Evolutionary Microbiology 48:187-194

Zhang Z-Y, Liu C, Zhu Y-Z, Wei Y-X, Tian F, Zhao G-P, Guo X-K (2012) Safety assessment of Lactobacillus plantarum JDM1 based on the complete genome International journal of food microbiology 153:166-170

Zhang Z, Schwartz S, Wagner L, Miller W (2000) A greedy algorithm for aligning DNA sequences Journal of Computational biology 7:203-214

\section{Tables}

Table 1. Primers used in this study

\begin{tabular}{|llll|}
\hline Gene & Forward $\left(\mathbf{5}^{\prime} \rightarrow \mathbf{3}^{\prime}\right)$ & Reverse $\left(\mathbf{5}^{\prime} \rightarrow \mathbf{3}^{\prime}\right)$ & Reference \\
\hline 16S rDNA & GAGTTTGATCCTGGCTCAG & AGAAAGGAGGTGATCCAGCC & $\begin{array}{c}\text { (Yoon et al. } \\
\text { 1998) }\end{array}$ \\
\hline GAPDH & TGGCAAAGTGGAGATTGTTGCC & AAGATGGTGATGGGCTTCCCG & NM_002046 \\
\hline IFN-beta & TTACACTGCCTTTGCCATCCAA & TCCCACGTCAATCTTTCCTCTT & NM_010510.1 \\
\hline IFN-gamma & ACTGGCAAAAGGATCGTGAC & GACCTGTGGGTTGTTGACCT & NM_008337.3 \\
\hline $\begin{array}{l}\text { MNV capsid } \\
\text { protein }\end{array}$ & CTCTCAGCCATGTACACCGG & TAGGGTGGTACAAGGGCAACAA & JQ237823.1 \\
\hline
\end{tabular}


Table 2. General genome information for L. johnsonii Byun-jo-01

\begin{tabular}{|l|l|}
\hline & L. johnsonii Byun-jo-01 \\
\hline Sequencing platforms & PacBio RS II / Illumina Hiseq2000 \\
\hline Assembler & PacBio SMRT Analysis 2.3.0 / Pilon (v1.21) \\
\hline Number of reads & 182,640 (PacBio) / 81,648,222 (Illumina) \\
\hline Genome coverage & 635 \\
\hline Genome size (bp) & $1,959,519$ \\
\hline G+C content (\%) & 34.7 \\
\hline Predicted CDS & 1781 \\
\hline Number of contigs & 1 \\
\hline Number of rRNA genes & 21 \\
\hline Number of tRNA genes & 77 \\
\hline N50 (bp) & $1,959,519$ \\
\hline
\end{tabular}

Table 3. Comparison of wholegenome similarity between $L$. johnsonii Byun-jo-01 and reference strains

\begin{tabular}{|lllll|}
\hline Strain & Coverage & Similarity & Origin & Accession \\
\hline NCC 533 & $93 \%$ & $99 \%$ & Homo sapiens & AE017198 \\
\hline BS15 & $90 \%$ & $99 \%$ & Homo sapiens & CP016400 \\
\hline N6.2 & $90 \%$ & $99 \%$ & Rattus & CP006811 \\
\hline UMNLJ22 & $80 \%$ & $98 \%$ & Meleagris gallopavo & CP021704 \\
\hline UMNLJ21 & $80 \%$ & $98 \%$ & Meleagris gallopavo & CP021703 \\
\hline DPC 6026 & $90 \%$ & $98 \%$ & Sus scrofa domesticus & CP002464 \\
\hline
\end{tabular}

Table 4. Measurement of food intake after administration of Lactobacillus

\begin{tabular}{|ll|}
\hline Strain & Food intake $(\mathbf{g})$ \\
\hline Negative control & $7.88 \pm 0.65$ \\
\hline L. paracasei ATCC 334 & $7.27 \pm 1.04$ \\
\hline L. johnsonii Byun-jo-01 & $7.28 \pm 0.87$ \\
\hline
\end{tabular}

Values are mean \pm SD for $n=3$ 
Table 5. Bacterial translocation from gastrointestinal tract to other tissues

\begin{tabular}{|lllll|}
\hline \multirow{3}{*}{ MRS agar } & Bacteria & Blood & Kidney & Liver \\
\cline { 2 - 5 } & Negative control & $0 / 3$ & $0 / 3$ & $0 / 3$ \\
\cline { 2 - 5 } & L. paracasei ATCC 334 & $0 / 3$ & $0 / 3$ & $1 / 3$ \\
\cline { 2 - 5 } B. johnsonii Byun-jo-01 & $0 / 3$ & $0 / 3$ & $0 / 3$ \\
\hline \multirow{3}{*}{ BHI agar } & Negative control & $0 / 3$ & $0 / 3$ & $0 / 3$ \\
\cline { 2 - 5 } & L. paracasei ATCC 334 & $0 / 3$ & $0 / 3$ & $0 / 3$ \\
\cline { 2 - 5 } & L. johnsonii Byun-jo-01 & $0 / 3$ & $0 / 3$ & $0 / 3$ \\
\hline
\end{tabular}

The value indicates the number of animals with translocation / total mice

\section{Figures}

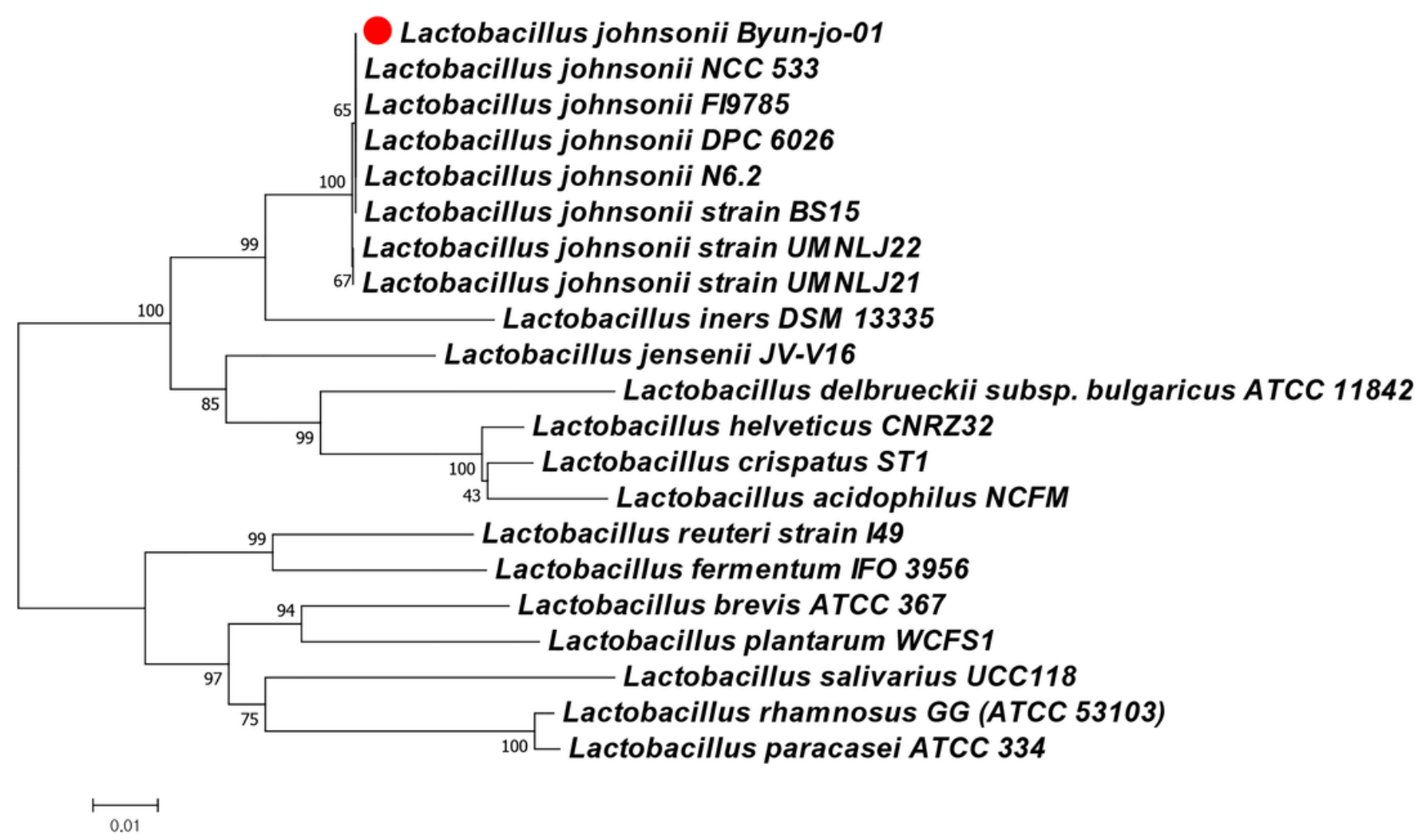

Figure 1

Phylogenetic relationships of L. johnsonii mouse isolates based on 16S rRNA sequences with reference sequences. Construction of the phylogenetic tree was performed using the Neighbor-Joining method. An average of 1500 nucleotides of 16S rRNA was aligned, and bootstrap values (1000 replicates) are 
indicated at the branch points. All values were greater than $40 \%$. Limosilactobacillus, Levilactobacillus, Lactiplantibacillus, Ligilactobacillus, Lacticaseibacillus, and Lactobacillus species used in this result were as follows: various L. johnsonii strains including L. johnsonii mouse isolate, L. fermentum, L. crispatus, L. brevis, L. paracasei, L. salivarius, L. plantarum, L. rhamnosus, L. iners, L. jensenii, L. acidophilus, L. delbrueckii, and L. helveticus. The scale represents 0.1 nucleotide substitutions per position.

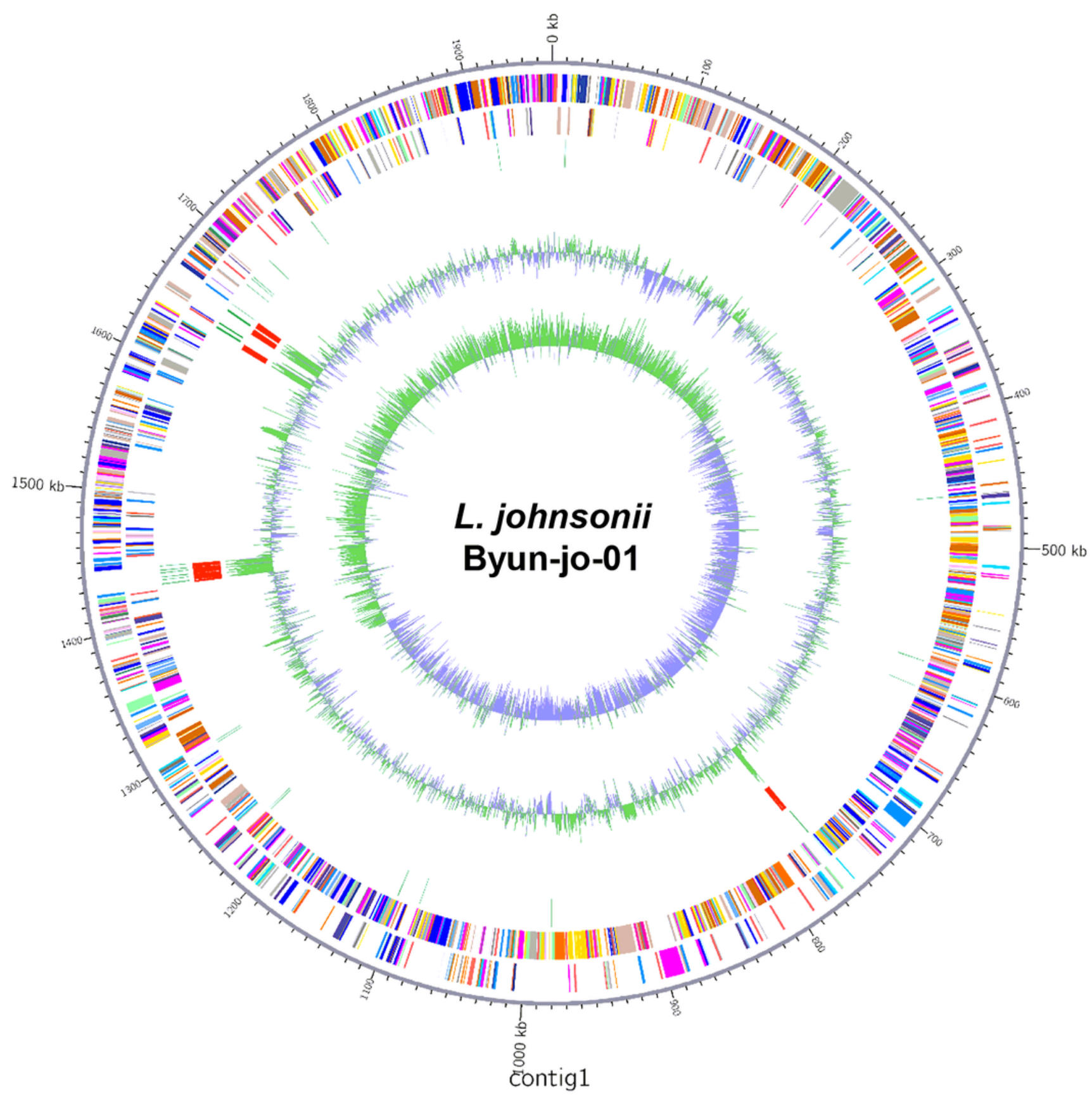

Figure 2

Circular representation of the L. johnsonii Byun-jo-01 genome. Representation of the chromosome map of Lactobacillus johnsonii Byun-jo-01. Marked characteristics are displayed from the outside to the center: 
CDS on forward strand, CDS on reverse strand, tRNA (light green), rRNA (red), GC content, and GC skew. This map was visualized using circos.

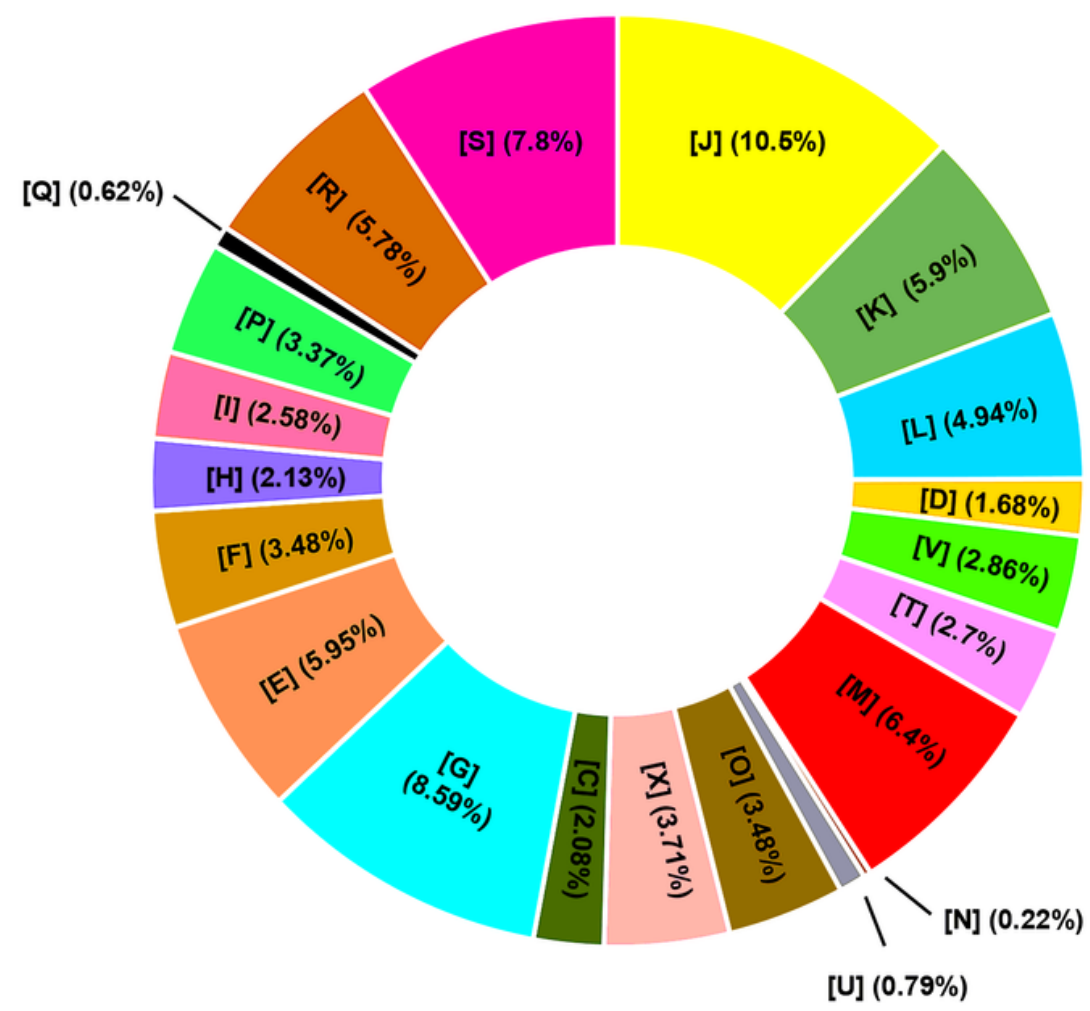

$\mathrm{J}$ Translation, ribosomal structure and biogenesis - K Transcription

- L Replication, recombination and repair

- D Cell cycle control, cell division

- $V$ Defense mechanisms

- T Signal transduction mechanisms

- M Cell wall/membrane/envelope biogenesis

- N Cell motility

= U Intracellular trafficking, secretion

- O Posttranslational modification, protein turnover

-X Mobilome: prophages, transposons

- C Energy production and conversion

- G Carbohydrate transport and metabolism

- E Amino acid transport and metabolism

- F Nucleotide transport and metabolism

- H Coenzyme transport and metabolism

- I Lipid transport and metabolism

- P Inorganic ion transport and metabolism

- $Q$ Secondary metabolites biosynthesis

- R General function prediction only

- S Function unknown

\section{Figure 3}

Clusters of orthologous group (COG) functional categories in L. johnsonii Byun-jo-01. Distribution of COG functional categories in L. johnsonii Byun-jo-01. Putative coding sequences were identified using PSIBLAST through NCBI COGs, 2014 version (http://www.ncbi.nlm.nih.gov/COG/) based on sequence homology. 


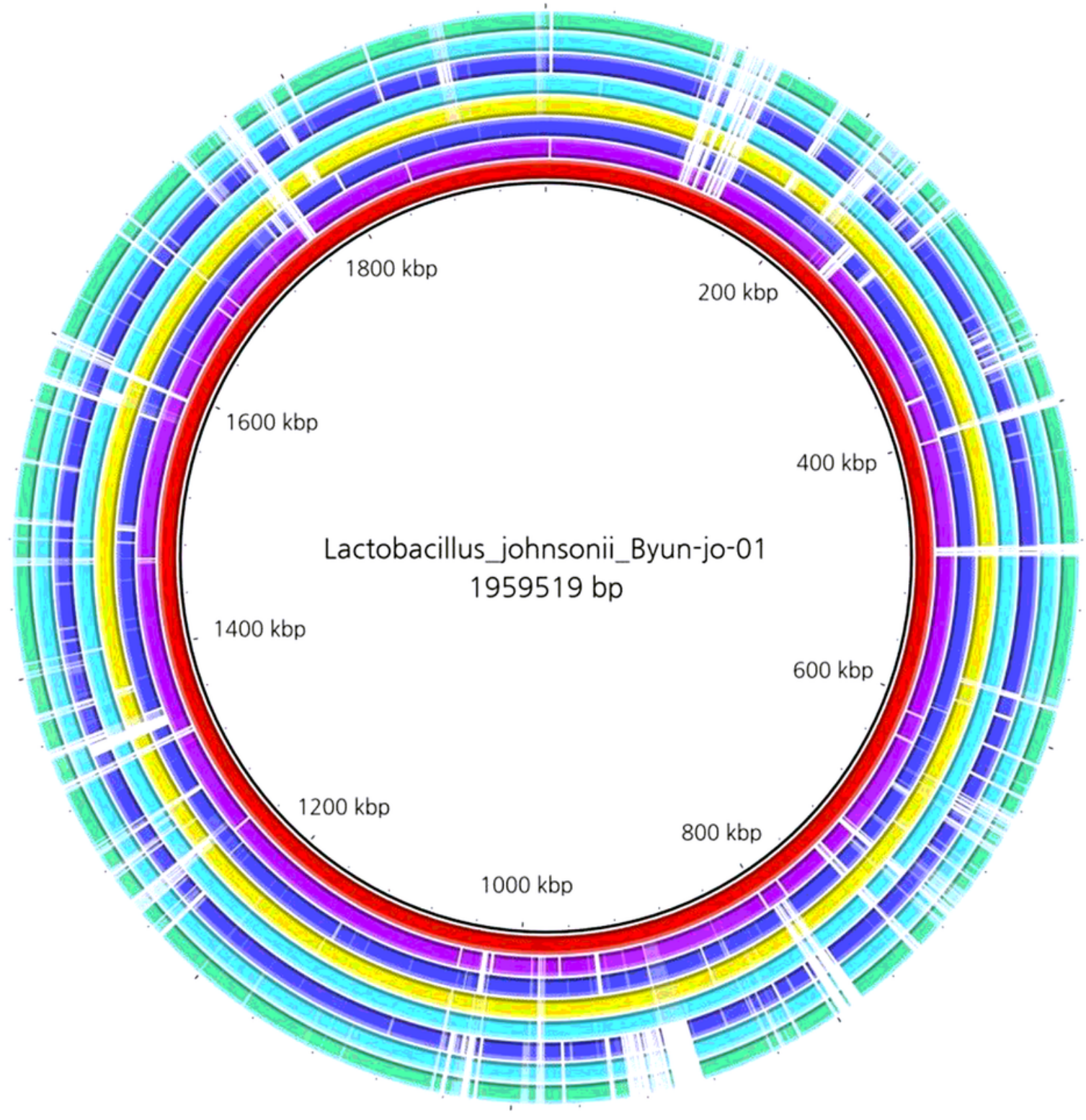

Byun-jo-01

$100 \%$ identity

$70 \%$ identity $50 \%$ identity

NCC533

$100 \%$ identity

$70 \%$ identity $50 \%$ identity

N6. 2

$\square 100 \%$ identity

- $70 \%$ identity $50 \%$ identity

BS15

-100\% identity

$70 \%$ identity $50 \%$ identity

DPC60206

$100 \%$ identity $70 \%$ identity $50 \%$ identity

Fl9785

$100 \%$ identity $70 \%$ identity $50 \%$ identity

UMNL21

[100\% identity

(7) $70 \%$ identity $50 \%$ identity

UMNLJ22

$100 \%$ identity $70 \%$ identity $50 \%$ identity

\section{Figure 4}

Similarity of the whole-genome sequence of L. johnsonii Byun-jo-01 with those of other L. johnsonii species. Comparison of the whole genome of L. johnsonii Byun-jo-01 was performed, and representation of the aligned circular map is shown using the BLAST Ring Image Generator (BRIG) with the default setting. To compare similarity, the genomes of L. johnsonii NCC533, L. johnsonii N6.2, L. johnsonii BS15, L. johnsonii DPC60206, L. johnsonii FI9785, L. johnsonii UMNLJ21, and L. johnsonii UMNLJ22 were obtained from the NCBI genome database (http://www.ncbi.nlm.nih.gov/genome). 


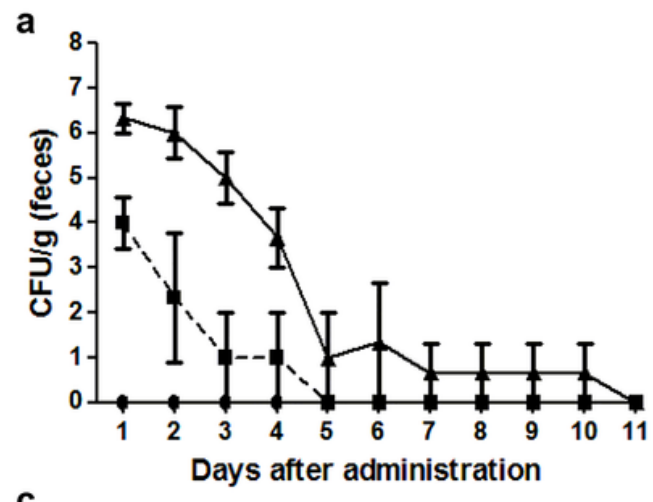

C

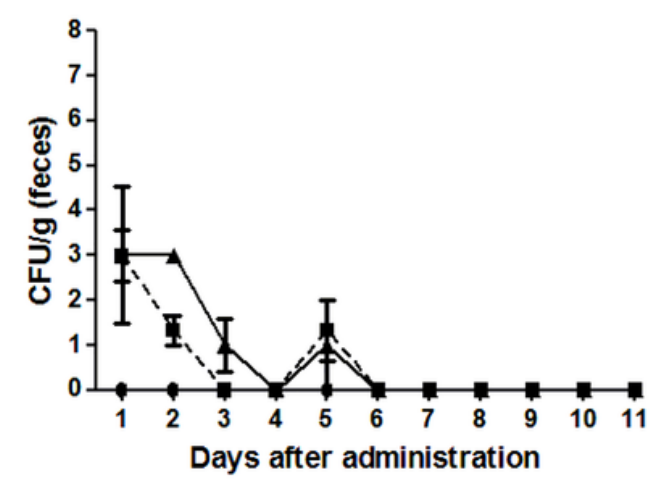

b

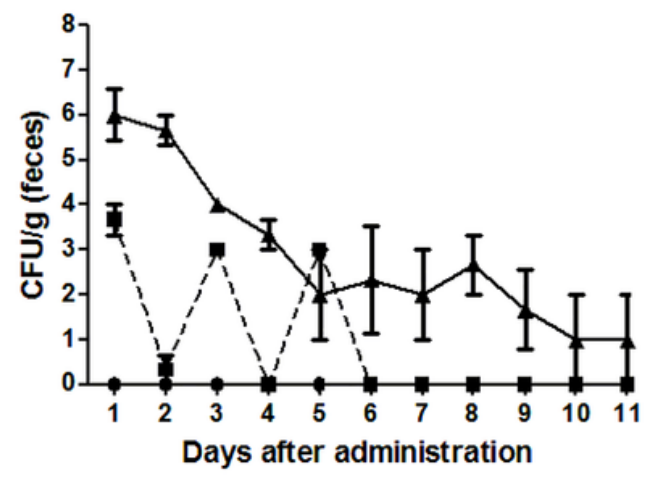

d

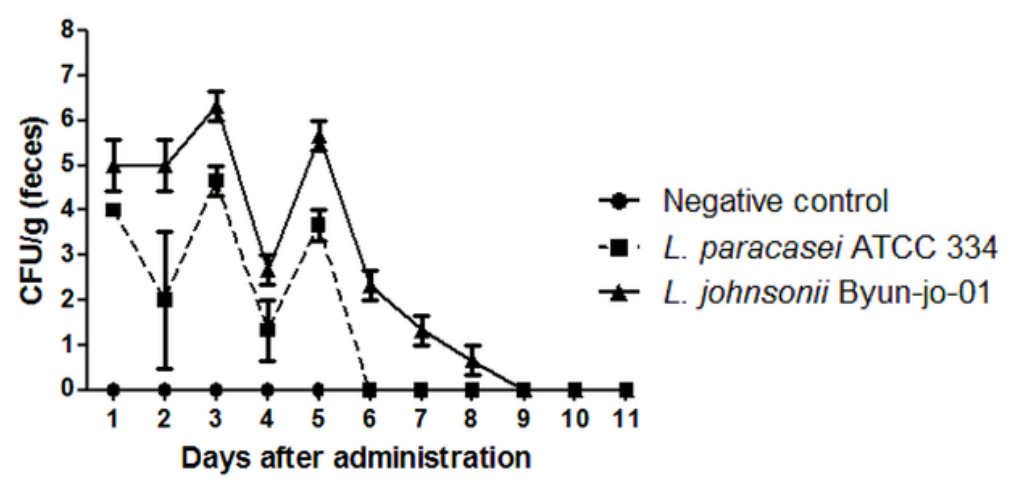

\section{Figure 5}

Persistence of L. johnsonii Byun-jo-01 in the gastrointestinal tract under various conditions. Under antibiotic treatment conditions, 108 CFUs of Lactobacillus strains (L. paracasei ATCC 334 and L. johnsonii Byun-jo-01) were administered once (a) and three times every two days (b). To analyze congenital persistence (the ability of L. johnsonii Byun-jo-01 to colonize the gut tissue in the absence of antibiotics), the Lactobacillus strains were administered once (c) and three times every two days (d). Data are presented as the mean \pm SEM. (O: Negative control, $\square$ : L. paracasei ATCC 334, $\mathbf{\Delta}$ : L. johnsonii Byunjo-01) 


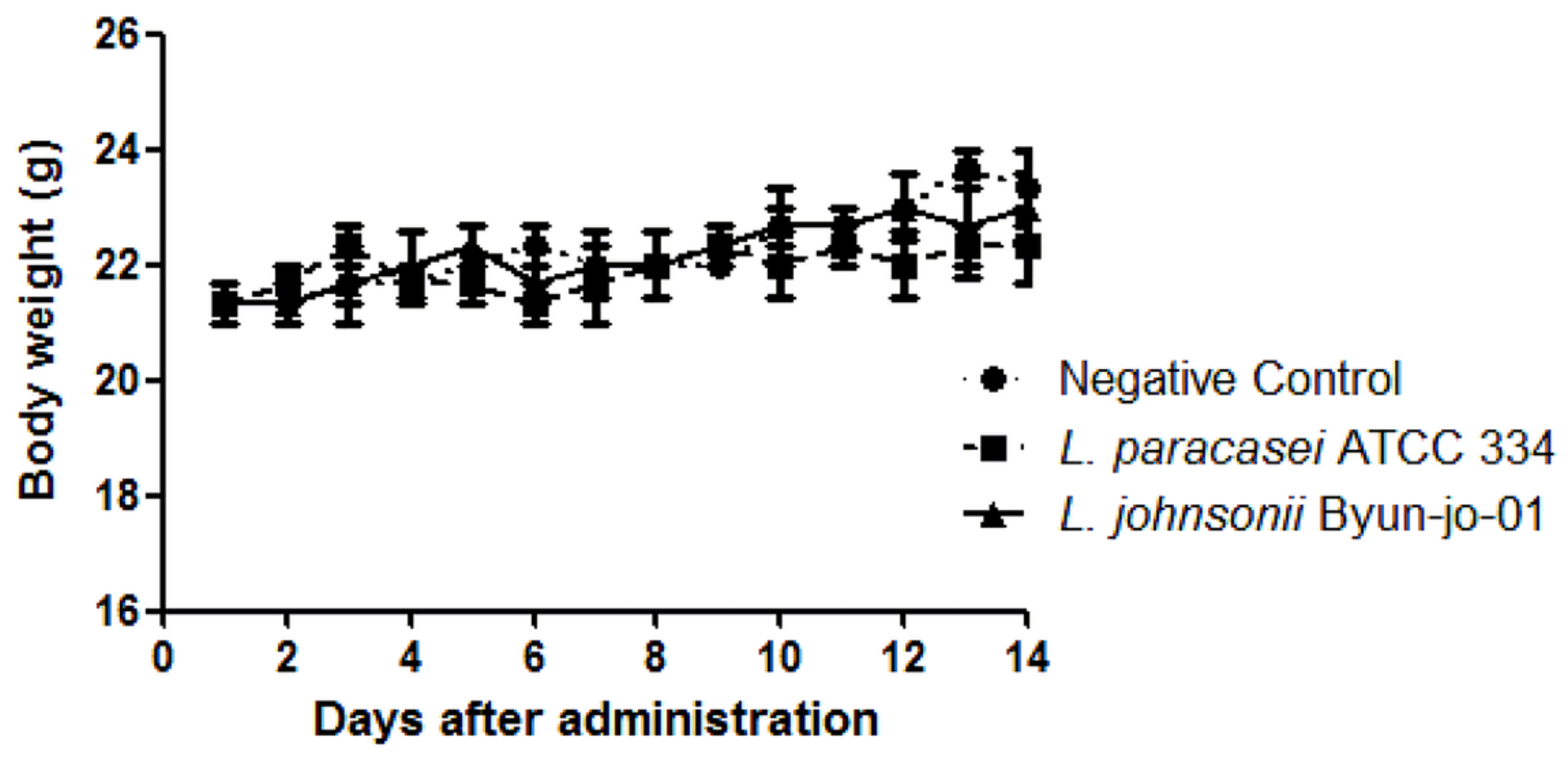

Figure 6

Comparison of weight change in mice treated with Lactobacillus and Lacticaseibacillus strains. L. paracasei ATCC 334 and L. johnsonii Byun-jo-01 were administered to mice daily for 2 weeks. During the administration period, the body weight of the mice was measured daily. Data are presented as the mean \pm SEM of $n=3$.
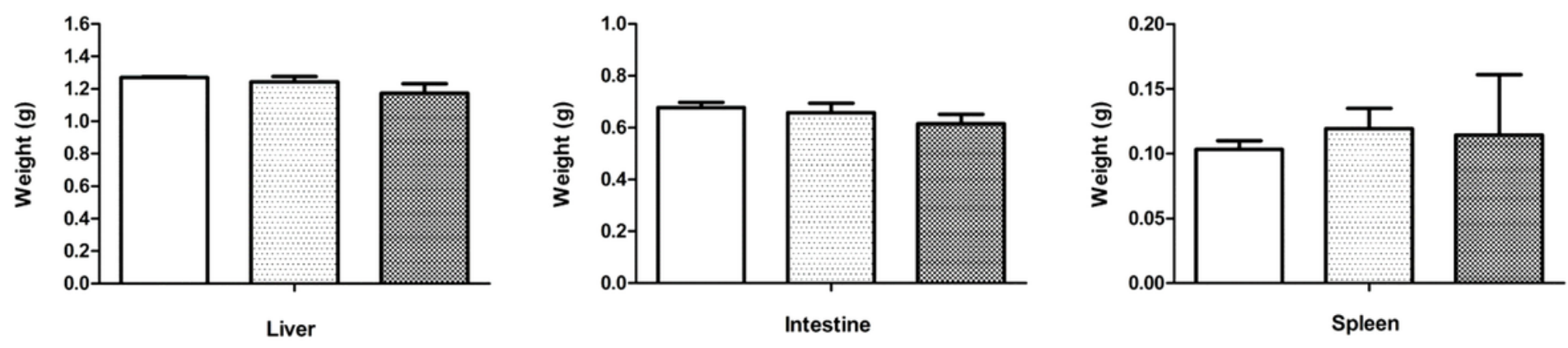

Figure 7

Comparison of organ weights among mice treated with Lactobacillus and Lacticaseibacillus strains. Mice treated with L. paracasei ATCC 334 as a positive probiotic were compared with mice treated with L. johnsonii Byun-jo-01. After 2 weeks, the mice were sacrificed, and their livers, spleens, and small intestines were harvested to measure weight. Data are presented as the mean \pm SEM of $n=3$. 
a

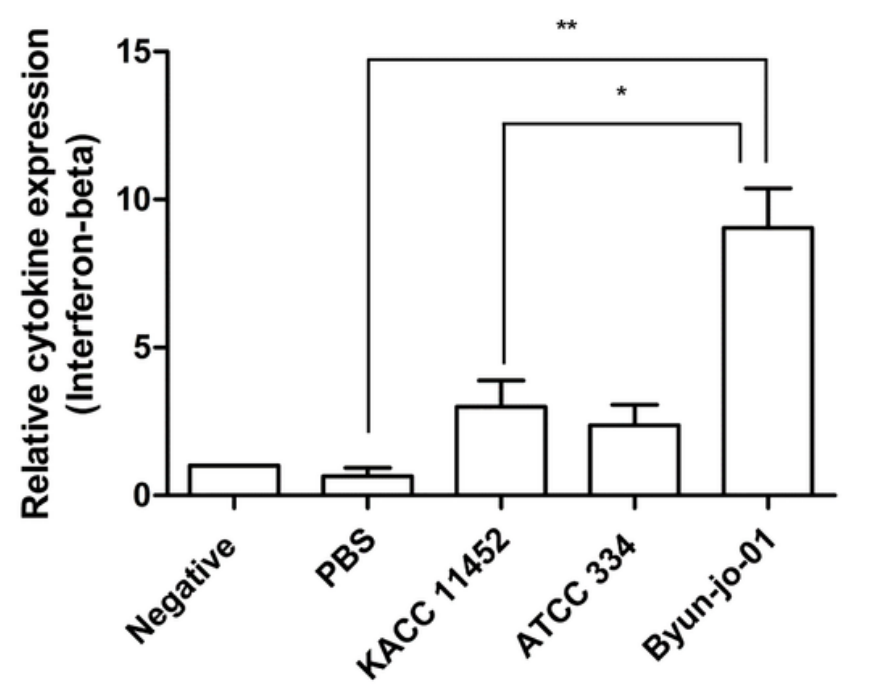

b

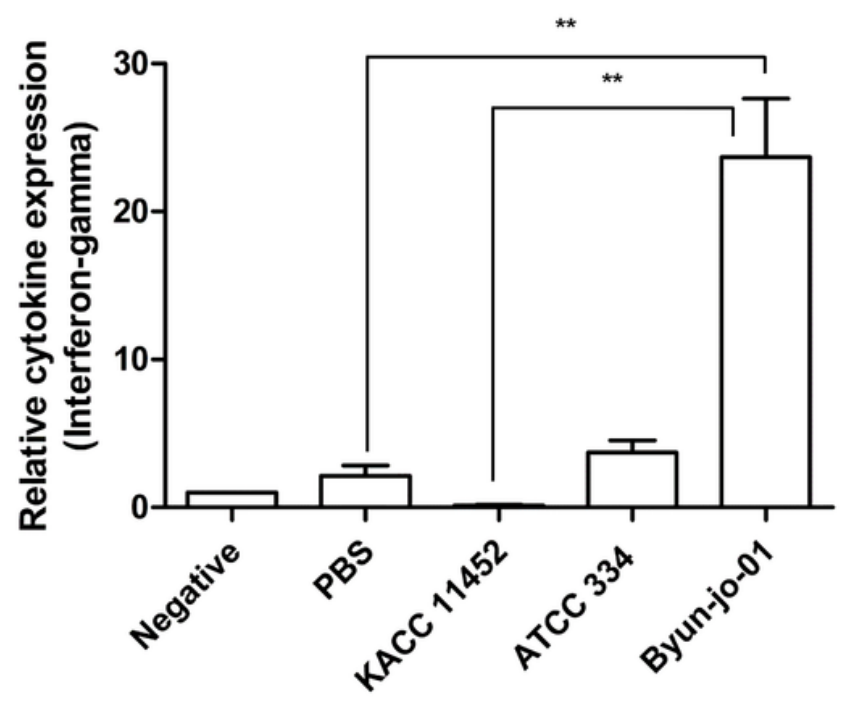

Figure 8

Immune boosting activity of L. johnsonii Byun-jo-01 in gut tissues. The expression levels of cytokines induced by Lactobacillus strains in gut tissues were measured using qRT-PCR. An additional control probiotic strain, L. reuteri KACC 11452 (originating from humans), was used to compare the immuneboosting activity of $L$. johnsonii Byun-jo-01. The relative mRNA expression of cytokines (a. IFN- $\beta, b$. IL-6, c. IFN- $y$, d. TNF-a) was quantified. GAPDH was used as an internal control, and the relative mRNA expression levels of the cytokines induced by Lactobacillus strains were calculated using the delta delta CT method. Data are presented as the mean \pm SEM of $n=3 .{ }^{*} p<0.05,{ }^{*} p<0.01$ 


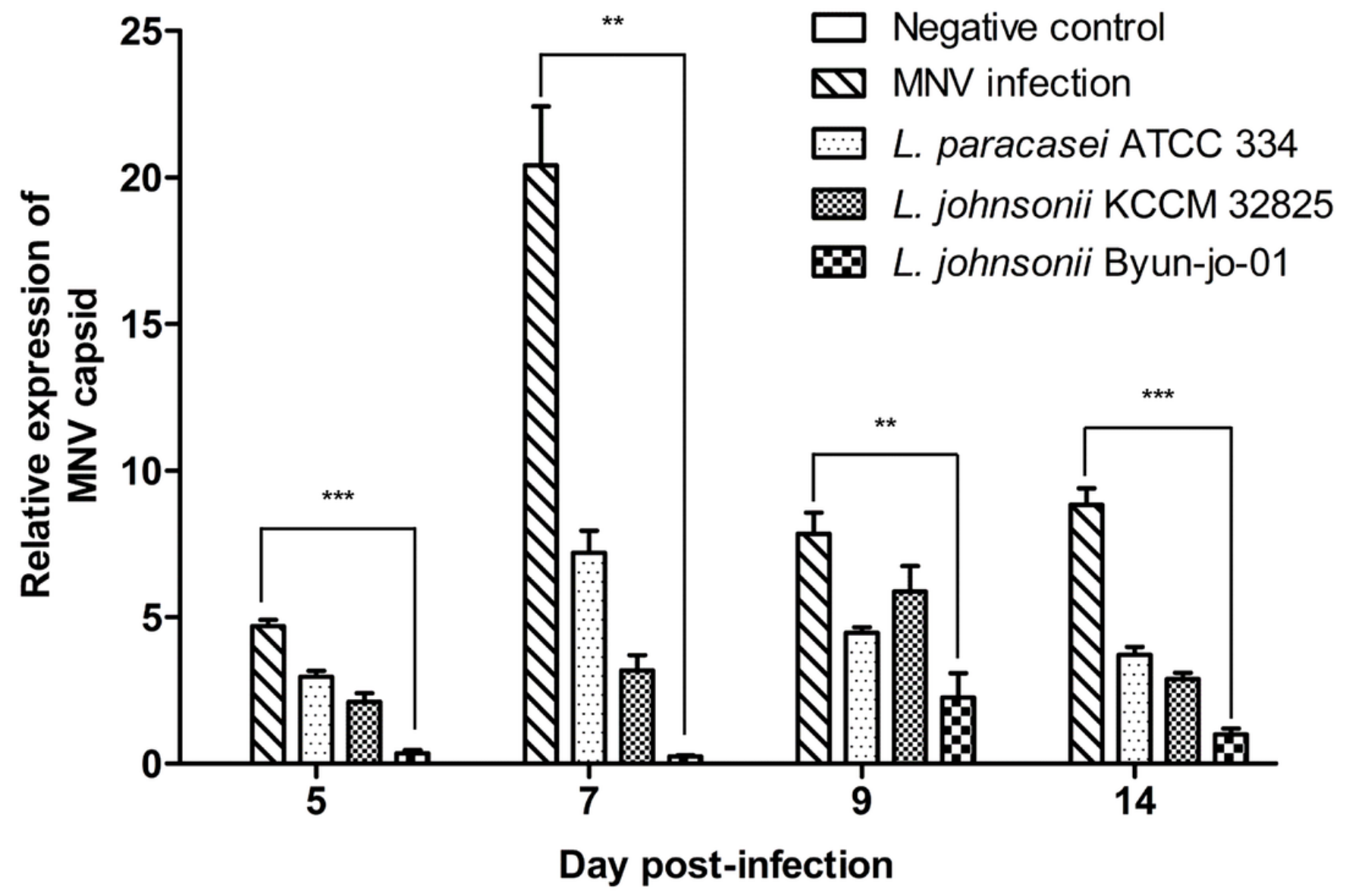

Figure 9

Antiviral efficacy of L. johnsonii Byun-jo-01 against murine norovirus strain CR6. To analyze the anti-MNV effect of Lactobacillus strains, they were administered to MNV-infected mice. Fecal samples collected on days 5, 7, 9, and 14 after infection were used for analysis. L. johnsonii KCCM32825 was used as another control probiotic. The relative expression of MNV capsid protein in fecal samples was analyzed. GAPDH was used as an internal control, and the relative mRNA level of capsid protein was calculated using the delta delta CT method. Data are presented as the mean \pm SEM of $n=3 .{ }^{* \star p} p<0.01,{ }^{* \star *} p<0.001$ 
Stress-related genes

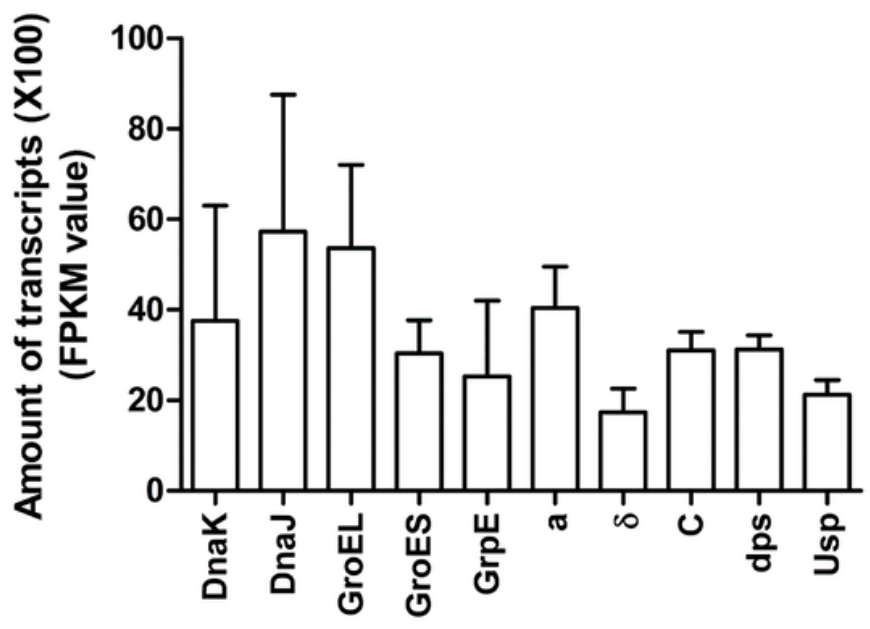

Virulence-related genes

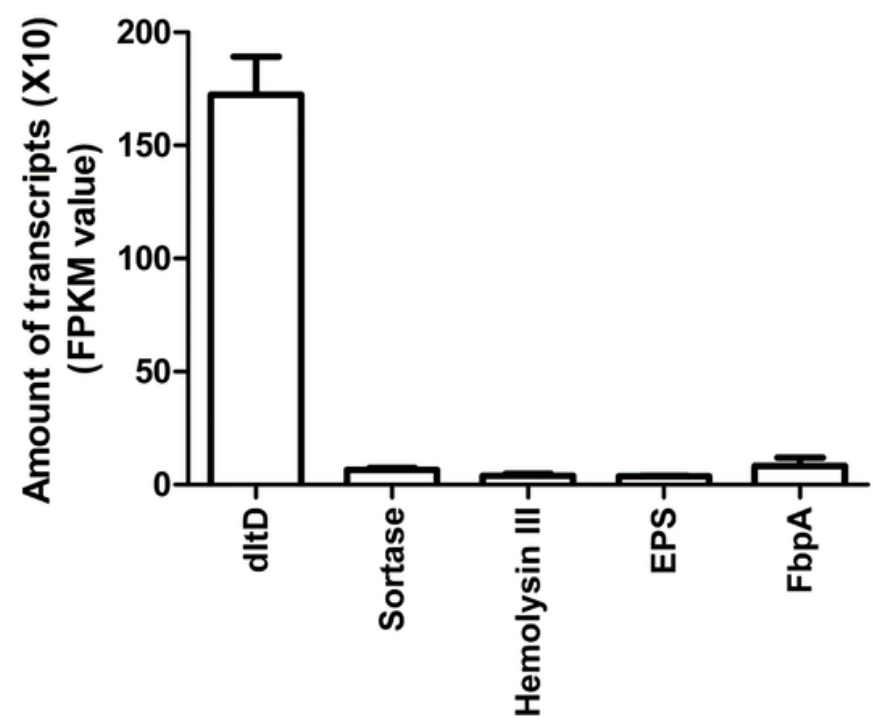

Figure 10

Screening of probiotic-specific markers through transcriptome data. To find probiotic-specific markers, a transcriptome analysis was conducted to identify various adaptation factors and probiotic factors. The expression level for each gene is indicated using the FPKM (Fragment Per Kilobase of transcript per Million mapped reads). Data are presented as the mean \pm SEM of $n=2$. 